Orinoquia, Enero-Junio 2020;24(1): 52-63

ISSN electrónico 2011-2629.

ISSN impreso 0121-3709.

http://doi.org/ 10.22579/20112629.601

\title{
¿Qué sabemos de los esteroides sexuales y las gonadotropinas en la reproducción de teleósteos neotropicales?
}

\author{
What do we know about sex steroids and gonadotropins regarding \\ Neotropical teleost reproduction?
}

\section{O que sabemos sobre os esteroides sexuais e as gonadotrofinas na reprodução de teleósteos neotropicais?}

\author{
Mónica T. Nieto-Vera ${ }^{1 *}$; José A. Rodríguez-Pulido² Agustín Góngora-Orjuela $^{3}$ \\ 1 Biol \\ 2 Biol, MSc, PhD, Grupo de investigación sobre reproducción y toxicología de organismos acuáticos (GRITOX), Instituto de \\ acuicultura, Universidad de los Llanos, Villavicencio,Colombia. \\ $3 \mathrm{MV}, \mathrm{MSc}, \mathrm{PhD}$ \\ * Grupo de investigación en reproducción y genética animal (GIRGA), Escuela de Medicina veterinaria y zootecnia, \\ Universidad de los Llanos, Villavicencio, Colombia. \\ Email:monica.nieto@unillanos.edu.co
}

Recibido: 29 de marzo de 2019

Aceptado: 24 de marzo de 2020

\begin{abstract}
Resumen
Esta revisión provee un resumen general de las investigaciones realizadas en los últimos 10 años acerca de los esteroides sexuales (17 -Estradiol, Testosterona, 11 Ketotestosterona y $17 \alpha, 20 \beta$-DHP), las gonadotropinas (Fsh y Lh) y sus principales reguladores (GnRH1-3 y GnlH1-3); y su respectiva función en la modulación de la reproducción. Específicamente sobre el eje Hipotálamo - Pituitaria - Gónada (HPG), en teleósteos modelo y neotropicales (Astyanax altiparanae, Steindachneridion parahybae, Salminus hillarii, Centropomus undecimalis). Mostramos que el papel regulador de éstos continúa siendo tema de discusión, pues varía en función de múltiples características, entre ellas, las estrategiareproductivas de la especie en cuestión y/o las condiciones en las que éstas son mantenidas durante el estudio. Históricamente, se ha descrito que la Fsh actúa en las fases tempranas de la reproducción, estimulando la biosíntesis de estradiol y testosterona necesarios para la gametogénesis; mientras la Lh está implicada en las fases finales del ciclo reproductivo (maduración final de los gametos y desove). Sin embargo, en especies con desarrollo asincrónico y desove múltiple, la Lh se produce durante todo el ciclo en paralelo con la Fsh, sugiriendo la necesidad de ambas hormonas en la gametogénesis y maduración final. Adicionalmente, investigaciones recientes en especies con comportamiento migratorio reproductivo, han evidenciado una disminución significativa en la expresión del ARNm de la subunidad $\beta$ de Lh (Ihb) cuando los individuos son mantenidos en cautiverio, lo que podría ocasionar alguna disfunción reproductiva bajo los actuales sistemas de cultivo, considerado hoy el gran problema de la acuicultura contemporánea. Desafortunadamente, los estudios de estos tópicos en especies suramericanas
\end{abstract}


son aún incipientes, por lo que se hace necesario centrar las investigaciones hacia el esclarecimiento del control neuroendocrino de la reproducción en especies nativas, más aún cuando estas especies son mantenidas en cautiverio.

Palabras clave: Gonadotropinas; esteroides sexuales; maduración gonadal; reproducción en peces

\begin{abstract}
This review summarises research over the last 10 years regarding sex steroids (17 $\beta$-estradiol, testosterone, 11 ketotestosterone and $17 \alpha, 20 \beta-\mathrm{DHP}$ ), gonadotropins (Fsh and $\mathrm{Lh}$ ), their main regulators (GnRH1-3 and GnlH1-3) and their functions in modulating reproduction. It focuses specifically on the hypothalamic-pituitary-gonadal (HPG) axis in model and Neotropical teleosts (Astyanax altiparanae, Steindachneridion parahybae, Salminus hillarii and Centropomus undecimalis). Their regulatory role continues to be a subject of discussion since it varies, depending on multiple characteristics such as the reproductive strategies of the species in question and/or the conditions in which these were maintained during the study period. It has been described that Fsh acts during the early stages of reproduction by stimulating the biosynthesis of estradiol and testosterone which are necessary for gametogenesis, whilst Lh is involved in the reproductive cycle's final stages (final gamete maturation and spawning). However, Lh occurs throughout the cycle, in parallel with Fsh, in species having asynchronous development and multiple spawning, suggesting the need for both hormones during gametogenesis and final maturation. Recent research regarding species having reproductive migratory behaviour has highlighted a significant decrease in Lh $\beta$ subunit (LH $\beta$ ) mRNA expression when individuals are kept in captivity; this may have led to reproductive dysfunction in current culture systems, considered today as being the greatest problem facing contemporary aquaculture. Unfortunately, studying such topics related to South American species is still in its early stages so research must be focused on clarifying native species' neuroendocrine control of reproduction, especially when these species are kept in captivity.
\end{abstract}

Keywords: Gonadotropin; sex steroid; gonadal maturation; fish reproduction.

\begin{abstract}
Resumo
Esta revisão apresenta um resumo geral das pesquisas realizadas nos últimos 10 anos sobre os esteroides sexuais (17 $\beta$-Estradiol, Testosterona, 11 Ketotestosterona e $17 \alpha, 20 \beta$-DHP), as gonadotrofinas (Fsh e Lh), seus principais reguladores (GnRH1-3 e GnlH1-3); e sua respetiva função na modulação da reprodução. Especificamente, sobre o eixo Hipotálamo - Pituitária - Gónada (HPG), em teleósteos modelos e neotropicais (Astyanax altiparanae, Steindachneridion parahybae, Salminus hillarii, Centropomus undecimalis). Mostra-se que o papel regulador deles continua sendo assunto de discussão, pois varia em função de múltiplas caraterísticas, por exemplo, as estratégias reprodutivas da espécie mencionada e/ou as condições experimentais. Historicamente, tem sido descrito que a Fsh atua nas fases iniciais da reprodução, estimulando a biossíntese de estradiol e testosterona necessários para a gametogéneses; enquanto a Lh está implicada nas fases finais do ciclo reprodutivo (maduração final das gametas e desova). No entanto, em espécies com desenvolvimento assíncrono e desova múltipla, a Lh é produzida durante o ciclo em paralelo com a Fsh, sugerindo a necessidade de ambos hormônios na gametogénese e a maduração final. Adicionalmente, pesquisas recentes em espécies com comportamento migratório reprodutivo evidenciam uma diminuição significativa na expressão do mRNA da subunidade $\beta$ da Lh (lhb) quando os indivíduos são mantidos em cativeiro, o que poderia resultar em alguma disfunção reprodutiva sob os atuais sistemas de cultura, sendo considerado hoje o grande problema da aquicultura contemporânea. Desafortunadamente, os estudos desses tópicos em espécies sul-americanas ainda são incipientes, pelo que é necessário concentrar as pesquisas para o esclarecimento do controle neuroendócrino da reprodução em espécies nativas, principalmente quando essas espécies são mantidas em cativeiro.
\end{abstract}

Palavras-chave: Gonadotrofinas; esteroides sexuais; maturação gonadal; reprodução em peixes

\section{Introducción}

Desde hace décadas se ha determinado que los esteroides juegan un papel clave en la coordinación de las actividades del eje hipotálamo-hipófisis-gónada, además de su actividad en el control en procesos como la diferenciación y determinación sexual, metabolismo, respuesta inmune, osmoregulación, ritmos circadianos, señalización de feromonas, apareamiento y reproducción (Tokarz et al., 2015; Nyuji et al., 2018; Wylie et al., 2018).
La reproducción involucra diversos procesos fisiológicos, controlados por órganos específicos como la glándula pineal, el hipotálamo, la hipófisis, el hígado y las gónadas Durante las etapas de crecimiento, los niveles hormonales involucrados en la reproducción, mantienen una producción relativamente baja. Una vez ocurre la madurez de la gónada, la actividad hormonal se ve influenciada por diferentes factores como la temperatura, el fotoperiodo, señales asociadas con las lluvias y otras aun poco estudiadas (Fig. 1) que pueden cambiar el entorno del pez provocando que se disparen los mecanismos activadores del proceso reproductivo (Miranda et al., 2013). 
Los procesos de desarrollo reproductivo en un pez maduro están controlados por dos clases de gonadotropinas hipofisiarias denominadas GtH I y GtH II. Dadas las similitudes en las secuencias entre la $\mathrm{GtH}-\mathrm{I}$ (peces) y la hormona folículo estimulante, Fsh (mamífero); y la GtH-II y la hormona luteinizante, Lh, se ha adoptado el uso de los términos Fsh y Lh para hacer referencia a las GtH-I y GtH-II, respectivamente (Planas, 1995; Yaron et al., 2003; Rocha, Arukwe y Kapoor, 2008). Los receptores de Fsh predominan en las células de la granulosa, en el folículo y en las células de Sertoli, mientras los receptores de Lh se asocian a las células de la teca en el folículo y a las células de Leydig fuera del ciste testicular (Planas, 1995).

La Fsh está involucrada principalmente en la vitelogénesis, al inducir la captura de Vitelogenina $(\mathrm{Vtg})$ que esta modulada por la síntesis folicular de estradiol $\left(E_{2}\right)$ (Tokarz, 2015). Mientras que la principal acción de la Lh es la regulación de la maduración del oocito, la ovulación y en el desove, se requiere de la síntesis previa de esteroides inductores de maduración (MIS, por sus siglas en inglés madurative inductor steroids) por lo que se conoce como hormona madurativa (Planas, 1995; Breton, Govoroun y Mikolajczyk, 1998; Nagahama y Yamashita, 2008; Valdebenito, Paiva y Berland, 2011).

La síntesis y secreción de ambas gonadotropinas está dada en respuesta a la estimulación de la hormona liberadora de gonadotropinas $(\mathrm{GnRH})$, la cual es suprimida por la hormona inhibidora de gonadotropinas (GnlH) (wylie, 2018). Cuando la síntesis de Lh y Fsh ocurre, son transportadas a las gónadas por el torrente sanguíneo y allí se desencadenan la esteroidogénesis gonadal y gametogénesis (Levavi-Sivan et al., 2010).

\section{Síntesis de esteroides sexuales}

La síntesis de andrógenos, principalmente 11 ketotestosterona (11-KT) y testosterona (T) se da por la acción de la Fsh, que favorece la proliferación de espermatogonias y finalmente la espermiogenesis (Schulz et al., 2010). Para la espermiación, la Lh provoca incrementos en los niveles plasmáticos de progestágenos o esteroides inductores de la maduración (MIS), 17 $\alpha$, 20 $\beta$-dihidroxi-4-pregne-3-ona (17 $\alpha, 20 \beta$-DP)

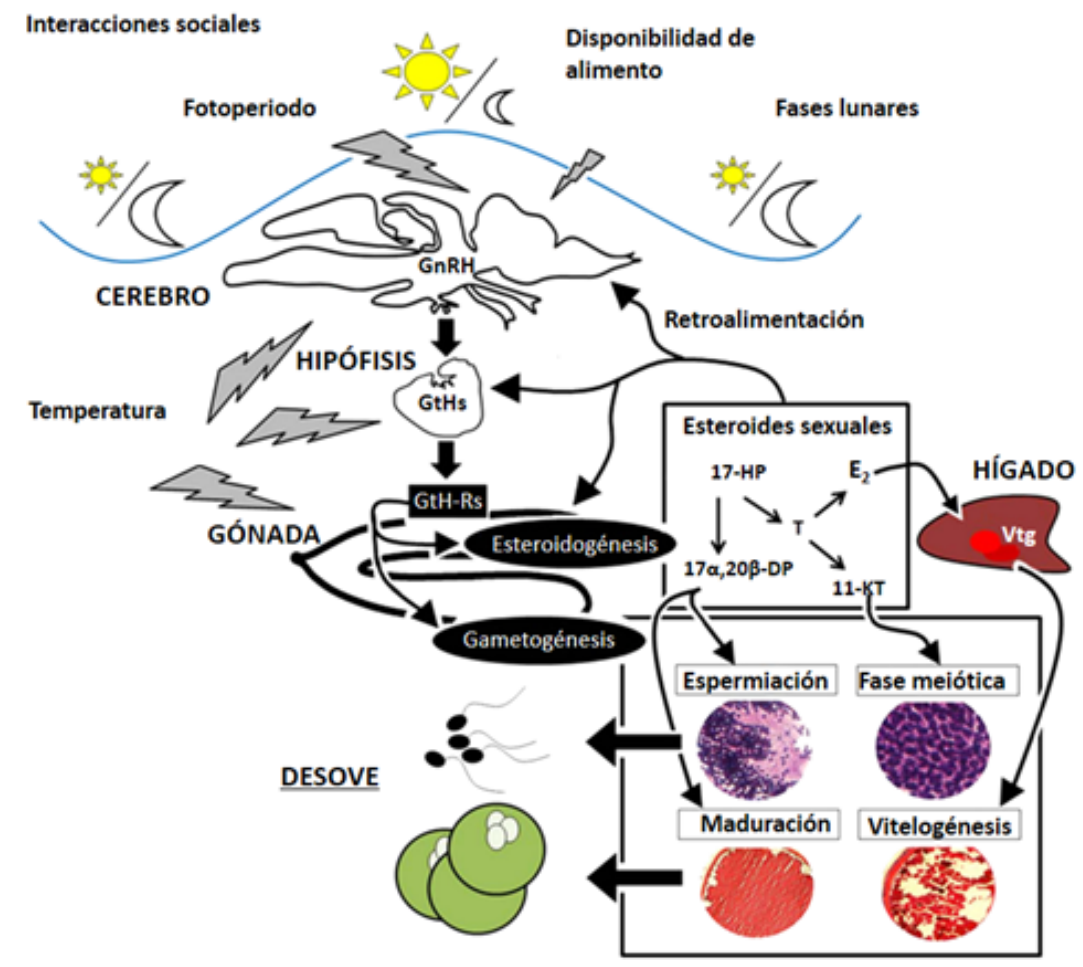

Figura 1. Influencia del ambiente en el control neuroendocrino de la reproducción, específicamente en el eje hipotalámico-pituitario-gonadal. Factores externos (fotoperiodo, fases lunares, disponibilidad de alimento, etc.) desencadenan en el cerebro del pez una serie de eventos hormonales que regulan las funciones reproductivas. $\mathbf{G n R}$ : hormona liberadora de gonadotropinas, GtHs: gonadotropinas (Fsh y Lh), GtH-Rs: receptores de gonadotropinas, 17-HP: 17 $\alpha$ hidroxiprogesterona, 17 $\alpha, 20 \beta-D P$ : 17 $\alpha$, $20 \beta$ dihidroxiprogesterona, T: testosterona, E2: 17 $\beta$-estradiol, 11-KT: 11 ketotestosterona, Vtg: vitelogenina. Fuente: modificado de: Miranda et al., 2013. 


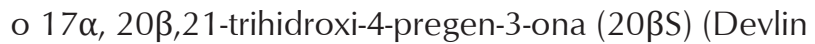
y Nagahama, 2002; Schulz et al., 2010).

En las hembras, La Lh y Fsh estimulan la producción de andrógenos a partir del colesterol en las células de la teca. Estos andrógenos, especialmente la testosterona pasa a las células de la granulosa en donde por acción de la P450 Aromatasa son transformados en estrógenos (Rocha, Arukwe y Kapoor, 2008; Tokarz et al., 2015). Los estrógenos, principalmente $17 \beta$-estradiol $\left(\mathrm{E}_{2}\right)$ son dirigidos al torrente sanguíneo y posteriormente captado por los receptores hepáticos, estimulando la síntesis hepática de vitelogenina (Vtg; Fig. 1) que se incorpora a los oocitos durante la vitelogenésis.

Si bien es sabido que la esteroidogénesis inicia a partir de un precursor común, el colesterol tras múltiples transformaciones mediadas por genes y enzimas ampliamente caracterizadas en muchas especies de teleósteos, vale la pena señalar que algunas de estas enzimas y genes sólo se han descrito para unos pocos teleósteos suramericanos. Ejemplo de esto es el gen $17 \beta$-hidroxiesteroide dehidrogenasa tipo 3 y tipo 1 (hsd17b3 y hsd17b1 respectivamente, Tokarz et al., 2013).

La hsd17b3 es una enzima esencial para la síntesis de 11-Ketotestosterona y ha sido caracterizada en 46 especies de teleósteos de las cuales se encuentra sólo un Siluriforme introducido (Pangasianodon hypophthalmus); mientras hsd17b1 que convierte la estrona inactiva ( $\left.E_{1}\right)$ en estradiol $\left(E_{2}\right)$ activo, se caracterizó en 51 especies las cuales incluye especies modelo como tilapia del Nilo, anguila japonesa, pez cebra, bacalao atlántico. Es por esto que la investigación para esclarecer estas rutas metabólicas aún no concluye y los estudios en silúridos deben iniciar, debido a su potencial como peces de cría en cautiverio (Tokarz et al., 2015; Chaube, Mishra y Singh, 2018).

\section{Hormona liberadora de Gonadotropinas (GnRH)}

Es un péptido hipotalámico modulador de la síntesis y liberación de gonadotropinas $(\mathrm{GtH})$ como la Fsh y Lh desde la glándula pituitaria (Zohar et al., 2010) y que consecuentemente estimula la síntesis de esteroides sexuales para el crecimiento gonadal y maduración (Nagahama Y., 1994; Nagahama y Yamashita, 2008; Zohar et al., 2010).

Se ha demostrado la existencia de tres genes parálogos de GnRH: GnRH1 o catfish GnRH (cfGnRH), GnRH2 o chicken GnRH (cGnRH) y GnRH3 o salmon GnRH (sGnRH) (Okubo y Nagahama, 2008), y al menos 14 isoformas en vertebrados. En teleósteos como Beloniformes, Perciformes y Siluriformes se han identificado GnRH1 y GnRH2, perdiendo la expresión de GnRH3, mientras $\mathrm{GnRH} 3$ ha sido identificada únicamente en Salmoniformes (Melamed y Sherwood, 2005; Okubo y Nagahama, 2008; Zohar et al., 2010). Sin embargo, existe evidencia que indica no una pérdida de la función de la GnRH en cuestión, sino que puede haber una compensación funcional que asumen los otros genes parálogos restantes. Es decir, para el caso de Siluriformes que han perdido el gen $\mathrm{GnRH} 3$, su función es compensada por las neuronas que expresan $\mathrm{GnRH} 1$ (Karigo y Oka, 2013).

GnRH1 y GnRH3 se expresan en el cerebro anterior ventral, el área preóptica telencefálica y el hipotálamo y son las responsables de la regulación en la reproducción, mientras $\mathrm{GnRH} 2$ está restringida a la parte dorsal del diencéfalo y parece tener una función neuromoduladora sobre el comportamiento sexual (desove, migración), agresivo (Melamed y Sherwood, 2005; Zohar et al., 2010) y aparentemente actúa como factor liberador de melatonina en la glándula pineal de teleósteos (Servil et al., 2010). Como regulador del comportamiento de alimentación, disminuye la ingesta de alimentos en el pez dorado (Carassius auratus) (Matsuda et al., 2008) y la expresión del ARNm de orexina hipotalámica que está asociada no sólo con el apetito y el comportamiento de alimentación (Hoskins, Xu y Volkoff, 2008), sino también con el de búsqueda/recompensa en peces (Volkoff, 2016).

Otros estudios realizados en C. auratus revelan el papel regulador de $\mathrm{GnRH} 2$ durante la espermatogénesis (específicamente durante la regresión gonadal), pues se ha demostrado que incrementa la fragmentación del ADN (apoptosis) en etapas tardías de la maduración testicular (Andreu-Vieyra, Buret y Habibi, 2005).

Investigaciones recientes en Silúridos suramericanos como Steindachneridion parahybae durante un ciclo reproductivo en cautiverio, se identificó la presencia y distribución de GnRH1 y GnRH2 en el cerebro (Fig. 2).

Los análisis permitieron ubicar las neuronas inmunoreactivas de $\mathrm{GnRH} 1$ en el hipotálamo medio basal y el área preóptica, cuyos axones estaban fuertemente inervando la glándula pituitaria y al Pars distalis proximalis (PDP; Honji et al., 2019). Los anteriores hallazgos, confirman la evidencia que en los Siluriformes, la GnRH1 estimula la secreción de GtHs. En este mismo estudio se encontró que los niveles de GnRH1 se mantuvieron altos en la etapa vitelogénica y la función moduladora en la síntesis y liberación de gonadotropinas fue a su vez confirmada, puesto que la vitelogénesis 


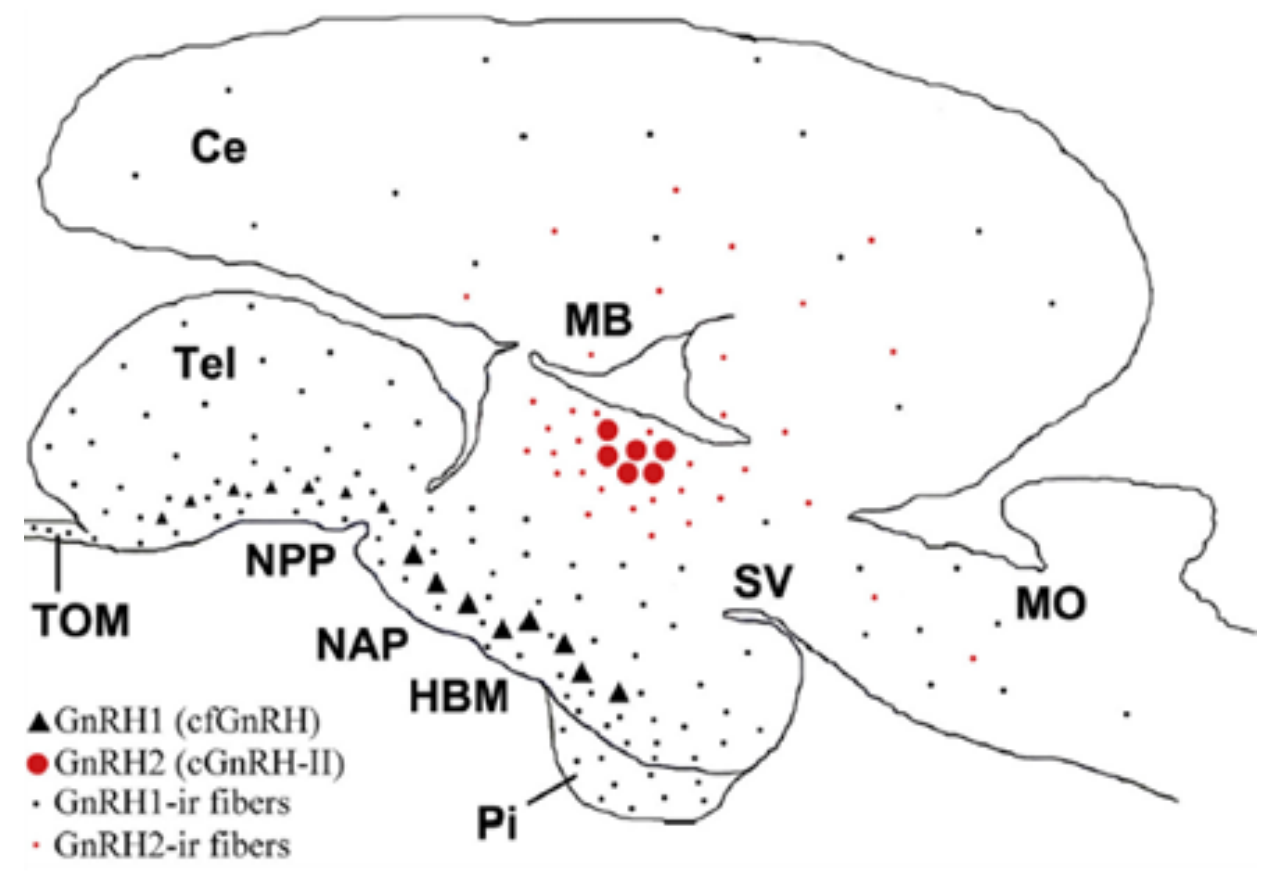

Figura 2. Diagrama sagital del cerebro de Steindachneridion parahybae que resume la distribución de GnRH 1-2 y sus respectivas fibras neuronales inmunoreactivas. Ce, cerebelo; HBM, hipotálamo basal medial; MB, cerebro medio; MO, médula oblongata; NAP, núcleo anterioris periventricular; NPP, núcleo preóptico periventricular; Pi, glándula pituitaria; SV, saccus vasculosus; Tel, telencéfalo; TOM, tracto olfativo medial. Tomada de (Honji et al., 2019).

está fuertemente controlada por la Fsh, en consecuencia la $\mathrm{GnRH}$ es sintetizada y liberada bajo cantidades suficientes de esteroides que modulan su actividad (Honji et al., 2019). Finalmente, los niveles de GnRH2 variaron poco en todo el ciclo reproductivo, aunque se encontraron elevados en la etapa de regresión, lo que sugiere un papel autocrino y/o paracrino en esta etapa de maduración ovárica caracterizada por oocitos atrésicos indicando absorción y/o apoptosis (Honji et al., 2019) similar a lo encontrado en los testículos de C. auratus (Andreu-Vieyra, 2005).

Por otro lado, estudios realizados en trucha arcoíris (Oncorhynchus mykiss) han demostrado el papel inhibitorio de la dopamina en la liberación de GnRH1 (Karigo y Oka, 2013; Hou et al., 2016). Además, bajo condiciones de cultivo en alta densidad, se demostró también la inhibición sobre la Fsh hipofisiaria, pues los niveles de expresión de ARNm presentaron una disminución significativa, seguida de aumentos marcados en los niveles de dopamina sérica (Hou et al., 2016); tal como había sido descrito en machos del pez dorado, bajo la aplicación del agonista dopaminérgico apomorfina (Yu y Peter, 1990).

Algunos neurotransmisores como la noradrenalina, serotonina (Yu, Rosenblum y Peter, 1991), neuropeptido
Y (Peng et al., 1993) y las kisspectinas y sus receptores GPR54 han demostrado un papel estimulador en la liberación de GnRH (Felip et al., 2009; Tena-Sempere et al., 2012; Ando, Shahjahan y Kitahashi, 2018). Éstos últimos (Kiss 1 y Kiss 2), son poderosos activadores en la liberación de gonadotropinas, ya que despolarizan las neuronas de GnRH1 y estimulan la liberación de Lh y Fsh desde la pituitaria al inicio de la pubertad (Karigo y Oka, 2013; Park et al., 2016; Rather et al., 2016).

\section{Hormona inhibidora de gonadotropinas (GnIH)}

Es un dodecapeptido identificado inicialmente en la codorniz japonesa (Coturnix japonica) que actúa sobre las neuronas de la glándula pituitaria y la $\mathrm{GnRH}$ (Ubuka, Son y Tsutsui, 2016) disminuyendo la liberación y síntesis de gonadotropinas en mamíferos (Ubuka et al., 2009; Thorson et al., 2014), aves (Tsutsui et al., 2000; Ciccone et al., 2004; Tsutsui, 2009), reptiles (Ukena et al., 2016) y anfibios (Chowdhury et al., 2011). Para mayor información sobre la GnlH de otros vertebrados, se recomienda al lector remitirse a (Tsutsui et al., 2018).

Adicionalmente, las neuronas de GnlH expresan el receptor de melatonina y la melatonina estimula la expresión de GnlH (Ubuka et al., 2005; Tsutsui, 2009) 
la cual sufre una retroalimentación negativa (Wylie, 2018), donde es importante resaltar el papel inhibidor de la melatonina en la reproducción de Teleósteos (Maitra et al., 2013).

A partir de estudios en Carassius auratus, se identificó la expresión de un neuropeptido hipofisiario LPXRF- $\mathbf{N H}_{2}$ (Leu-Pro-X-Arg-Phe, donde $\boldsymbol{X}$ puede ser Leucina o Glutamina) que podía tener efectos sobre la glándula pituitaria regulando así la liberación de las hormonas hipofisiarias (Sawada et al., 2002). Mediante técnicas de Hibridación in situ se identificó su ARNm en el núcleo posterior periventricular del hipotálamo y mediante la técnica de ELISA, se determinó que las mayores concentraciones se encontraban en el diencéfalo y teléncefalo (Sawada et al., 2002). Los cuerpos celulares y los axones inmunoreactivos se localizaron a su vez en el núcleo posterioris periventricularis (NPPv) y en el núcleo lateral tubelar pars posterioris (NLTp) del diencéfalo, respectivamente. Estos hallazgos, permitieron sugerir su participación en la regulación de hormonas, pues dichas zonas del diencéfalo se han visto involucradas en el control de las funciones hipofisiarias (Sawada et al., 2002). Por último, Dada la homología del carbono terminal de este neuropeptido y la $\mathrm{GnlH}$ se consideró que el neuropeptido encontrado en C. auratus, era un fragmento de la hormona inhibidora de gonadotropinas (Sawada et al., 2002; Tsutsui, 2009; Tsutsui et al., 2018) por lo que esta hormona estaría presente también en peces.

Si bien los efectos de la GnIH en teleósteos aún es tema de investigación, controversia y debate, hay acuerdo sobre el papel de esta hormona en la regulación sobre el eje hipotálamo - pituitaria - gónada (HPG). En hembras de C. auratus inyectadas intraperitoneal con una mezcla de péptidos sintéticos de GnIH2 (AKSNINLPQRF-NH 2 ) y GnIH3 (SGTGLSATLPQRF-NH 2 ), se encontró una disminución significativa de los niveles de ARNm de gnrh3 y la subunidad $\beta$ de Fsh (fshb) en la glándula pituitaria, a la vez que $\mathrm{GnlH} 2$ disminuyó significativamente los niveles de ARNm de la subunidad $\beta$ de $L h(l h b)$. Sin embargo, cuando se aplicó $\mathrm{GnIH} 3$ a células primarias de cultivo de pituitaria se observó inhibición en la síntesis de lhb y fshb estimulada por GnRH (Qi et al., 2013).

Hallazgos similares fueron reportados para C. auratus inyectados intraperitonealmente con $\mathrm{GnIH}$ de pez cebra (Danio rerio), donde también se observó una disminución significativa de los niveles de Lh en plasma (Zhang et al., 2010) demostrando así la posible regulación negativa de la GnlH en teleósteos.
En carpa común (Ciprinus carpio) especie importante en la acuicultura, también se ha estudiado el rol negativo del sistema $\mathrm{GnlH} / \mathrm{GnlHR}$ (receptores de $\mathrm{GnlH}$ ) sobre la expresión de gonadotropinas. Hembras maduras inyectadas intraperitonealmente con el péptido $\mathrm{GnlH} 3$ con dos dosis diferentes (10 $\mathrm{\mu l} / \mathrm{kg}$ y $100 \mathrm{\mu l} / \mathrm{kg})$, presentaron cambios en los niveles de expresión de ARNm de las gonadotropinas (Peng et al., 2016). Los mismos efectos fueron encontrados in vitro bajo la

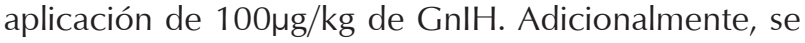
detectaron bajos niveles de expresión de $\mathrm{ARNm}$ de gnih en los meses previos al desove y un aumento significativo después del desove (Peng et al., 2016).

No obstante, se han reportado resultados diferentes en el salmón rojo (Oncorhynchus nerka) y tilapia (Oreochromis niloticus). Para el primer organismo, utilizando cultivos primarios de pituitaria e incubándolos bajo dosis diferentes de tres péptidos de $\mathrm{GnIH} 1-3$ de pez dorado (C. auratus; gfLPXRFa-1, -2, y -3), se encontró un efecto en la liberación de Fsh, Lh y la hormona de crecimiento $(\mathrm{GH})$ dependiente de la dosis (Amano et al., 2006). En el segundo organismo se suministró GnIH2 (LPXRFa-2) a cultivos primarios de pituitaria de tilapia y a hembras reproductivas mediante inyecciones intraperitoneales, encontrando efectos positivos en la liberación de Fsh y Lh in vivo e in vitro tras las primeras dos horas de la inyección (Biran et al., 2014).

\section{Hormona folículo estimulante (Fsh)}

Hormona pituitaria encargada de estimular la biosíntesis de esteroides sexuales como estradiol y testosterona, que consecuentemente regulan algunas etapas de la ovogénesis y espermatogénesis (Swanson, Dickey y Campbell, 2003). En los machos de salmón atlántico (Salmo salar), el incremento de ARNm de fshb en la pituitaria y de 11-KT en el plasma sanguíneo desencadenan la espermatogénesis (Melo et al., 2015).

En Salmoniformes que presentan un desarrollo ovárico sincrónico o sincrónico por grupos, se ha demostrado que la Fsh actúa en las fases tempranas del ciclo reproductivo (de Jesus et al., 2017). En trucha arcoíris (Oncorhynchus mykiss), la expresión del ARNm de la fsh hipofisiaria aumenta gradualmente conforme avanzan las fases iniciales del desarrollo ovocitario, confirmando el papel de esta hormona en el crecimiento del ovocito (vitelogenesis) (Hou et al., 2016).

Sin embargo, en Characiformes, Cypriniformes, Perciformes y Pleuronectiformes que presentan desarrollo ovárico asincrónico, se encontró que los niveles de transcripción de ARNm de fsh aumentaron también durante las fases finales del ciclo (Kajimura et al., 
2001). En hembras de Astyanax altiparanae cuyo desarrollo ovárico es asincrónico y presenta múltiples desoves, los niveles de ARNm de fshb aumentaron durante el predesove y se observó un segundo aumento postdesove. Ésto podría estar asociado con la proliferación de oogonias, pues se observó una gran cantidad de oocitos perinucleares, estimulando así, el desarrollo de nuevas cohortes de oocitos para un nuevo ciclo reproductivo (de Jesus et al., 2017).

Algo similar fue encontrado en Salminus hillarii, characiforme suramericano que tiene un comportamiento migratorio reproductivo y desarrollo ovárico sincrónico con un único desove. En hembras silvestres, se observaron altos niveles de expresión de ARNm de fshb durante la etapa previtelogenica que disminuyeron significativamente durante la maduración avanzada y subsecuentemente incrementaron durante la regresión (Moreira et al., 2015). Sin embargo, en hembras mantenidas en cautiverio, estos niveles fueron extremadamente bajos en todas las etapas de desarrollo ovocitario y en consecuencia, los niveles séricos de $E_{2}$ también se mantuvieron bajos, pudiendo tener una influencia en el menor reclutamiento de ovocitos en estas hembras (Moreira et al., 2015).

\section{Hormona luteinizante (Lh)}

Esta hormona pituitaria está implicada principalmente en las fases finales de ciclo reproductivo como la meiosis de las células germinales, la maduración final de los ovocitos, la ovulación y el desove a través del progestágeno 17 $\alpha, 20 \beta$-DHP (Swanson, Dickey y Campbell, 2003; Lubzens et al., 2010). Esto ha sido observado en hembras de trucha arcoíris cuyo ovario se encontraba en estadio IV de maduración (primer estadio vitelogénico). En este punto, se encontró que la expresión del ARNm de Ih tuvo sus picos más altos al igual que las progestinas como Progesterona ( $\left.\mathrm{P}_{4}\right)$ y $17 \alpha, 20 \beta$-DHP, principal hormona inductora de la maduración (Hou et al., 2016).

En algunas especies con desarrollo ovárico asincrónico y desove múltiple, los niveles de Lh aumentan en paralelo con los de Fsh durante el desarrollo gonadal (Mateos et al., 2003), como fue encontrado en machos de lubina europea (Dicentrarchus labrax), donde los niveles de ARNm de $f s h b$ y lhb aumentaron a medida que se desarrollaba la espermatogénesis y disminuyeron post-espermiación, sugiriendo la necesidad de ambas hormonas en la gametogénesis y maduración gonadal (Mateos et al., 2003).

Las hembras de Astyanax altiparanae, mantenidas en cautiverio presentaron niveles constantes de ARNm de lhb durante todo el ciclo reproductivo, aunque se observó una disminución significativa durante el pre-desove, evidenciando un posible fallo en la síntesis de esta hormona necesaria para la maduración final de los ovocitos y el desove (de Jesus et al., 2017). Estos resultados podrían explicar la disfunción reproductiva de esta especie en cautiverio, sin embargo no hay estudios suficientes que validen esta hipótesis.

En otro Characiforme, Salminus hilarii, los niveles de expresión de lhb pituitaria tuvieron un incremento de la fase previtelogénica (PV) a maduración avanzada (AM) y se mantuvieron durante la regresión (RGG) en hembras silvestres. Aunque estos niveles fueron más bajos en hembras en cautiverio, hubo un incremento de PV a AM y se mantuvieron en RGG (Moreira et al., 2015). Pese a esto, las hembras no lograron desovar por lo que la disfunción reproductiva que se presenta en los animales en cautiverio, no se puede relacionar únicamente con la falta de síntesis de Lh; sino que podría existir influencia de otros aspectos comportamentales, ambientales y fisiológicos propios de la especie sobre la incapacidad de desove, que hasta el momento no han sido probados.

\section{$17 \beta$-Estradiol $\left(E_{2}\right)$}

El estradiol estimula la síntesis hepática de la vitelogenina $(V \operatorname{tg})$ y con ello la maduración final de oocitos, metabolizando lípidos (etapa lipogénica) hacia el ooplasma (Devlin y Nagahama, 2002; Rocha, Arukwe y Kapoor, 2008). El estradiol, se encuentra presente también en machos aunque en concentraciones más bajas niveles y se ha evidenciado que en éstos regula la renovación de las espermatogonias (Miura et al., 1999) y la expresión de genes importantes en la espermatogénesis y esteroidogénesis (Schulz et al., 2010).

Estudios recientes en Silúridos asiáticos demostraron altos niveles de $\mathrm{E}_{2}$ en la vesícula seminal y testículo de Heteropneustes fossilis, debido a la presencia de la aromatasa y sus receptores para la expresión de estrógenos (ER por sus siglas en inglés, estrogen receptor) en las células somáticas testiculares (Chaube, Mishra y Singh, 2018). La influencia del $E_{2}$ en la espermatogénesis ha sido demostrada en el bagre de canal (Ictalurus punctatus) donde se encontraron genes de ER $\alpha$ y ER $\beta$ en los epitelios germinales y no germinales del testículo, cuya interacción podría tener influencia en el desarrollo de los gametos masculinos (espermatogénesis) y la fertilidad (Wu et al., 2001).

Adicionalmente, perfiles hormonales realizados en hembras reproductoras (de 2 a 3 años de edad) de bagre de canal, mostraron cambios temporales en los ni- 
veles de $E_{2}$ cuya concentración aumentó en los meses de predesove. Este aumento se podría constituir como un inductor para la producción de Vtg, la cual aumentó en el plasma a medida que las concentraciones de E2 también aumentaban, corroborando el importante rol de este esteroide en la vitelogénesis (Barrero et al., 2007).

Respecto a Salmoniformes, en la trucha arcoíris (Oncorhynchus mykiss) mediante Radioinmunoensayo (RIA) y ELISA, se encontró que los niveles de $E_{2}$ sérico aumentan conforme al aumento del índice gonadosomático, el cual está directamente asociado con crecimiento ovárico (Saborido-Rey, 2008; Hou et al., 2016). Además se ha demostrado que bajo condiciones de cría en alta densidad $\left(36-49.3 \mathrm{~kg} / \mathrm{m}^{3}\right)$, los niveles de $E_{2}$ disminuyen significativamente en contraste con grupos control criados a una densidad estándar (4,6 - $8.6 \mathrm{Kg} / \mathrm{m}^{3}$ ) (Hou et al., 2016). Esto puede deberse al estrés crónico (aumento de cortisol) generado bajo este tipo de cultivo y el consecuente papel regulador del cortisol en la vitelogénesis (Mommsen, Vijayan y Moon, 1999; Hou et al., 2016).

Para finalizar, niveles de $\mathrm{E}_{2}$ medidos en machos de róbalo blanco (Centropomus undecimalis) criados en condiciones controladas mostraron concentraciones más altas en comparación con los criados en condiciones naturales. Lo que se puede explicar con base a la relación directa entre la temperatura y la actividad de la aromatasa gonadal y como consecuencia, en un aumento en los niveles de $\mathrm{E}_{2}$ en plasma sanguíneo (Passini et al., 2019).

\section{Testosterona ( $\mathrm{T}$ ) y 11-Ketotestosterona (11-KT)}

En los teleósteos, la 11-Ketotestosterona (11-KT) es el andrógeno más importante, comparado con la Testosterona $(\mathrm{T})$, para estimular el desarrollo de los testículos, la formación de gametos (espermatogénesis) y el comportamiento reproductivo de los machos (Borg, 1994; Schulz et al., 2010). La T presente en las hembras, sirve como substrato para la síntesis de estrógenos como $\mathrm{E}_{2}$ por acción de la enzima aromatasa (Rocha, Arukwe y Kapoor, 2008). La función de estos andrógenos aromatizables ha sido ampliamente estudiada, pero aquí nos remitiremos a los estudios de las dos últimas décadas.

A partir de experimentos en machos inmaduros de bagre africano (Clarias gariepinus) a los cuales se les implantaron pellets que contenían 11-KT y $\mathrm{T}$, se pudo demostrar que la 11-KT (pero no la T) estimulaba la espermatogénesis y la $\mathrm{T}$ (pero no la 11-KT) elevaba los niveles de la gonadotropina hipofisiaria Lh (Cavaco et al., 2001). Sin embargo, los perfiles hormonales de
T y 11-KT determinados en róbalos blancos (Centropomus undecimalis), demostraron que las concentraciones de estos esteroides están asociadas y aumentan significativamente a medida que los individuos alcanzaban la pubertad, es decir que a medida que avanza la espermatogénesis, también lo hacía el crecimiento testicular (Passini et al., 2019). En individuos inmaduros de Salmón del Atlántico (Salmo salar), una inyección con Testosterona $(75 \mu \mathrm{l} / \mathrm{g})$ y Adrenosterona (25 $\mu \mathrm{l} / \mathrm{g}$; ésta es convertida in vivo en 11-KT), provocó un aumento elevado de andrógenos en el plasma sanguíneo, estimulando directamente la diferenciación de las espermatogonias hasta cinco veces que en aquellos individuos que no fueron inyectados (Melo et al., 2015). Resultados coincidentes fueron encontrados en machos del capitán de la sabana (Eremophilus mutisii), silúrido de migraciones cortas, cuyos niveles de $T$ aumentaron durante la maduración previa al desove, coincidentes con los periodos de reproducción de la especie y el índice gonadosomático (Landines et al., 2017).

Adicionalmente, los niveles de Testosterona en el plasma sanguíneo de bagres de canal (hembras) fueron más altos $(0.22 \mathrm{ng} / \mathrm{ml})$ casi al mismo tiempo en que los niveles de vitelogenina iniciaron su aumento, lo que pudo deberse al uso de testosterona en la aromatización a $E_{2}$ (Barrero et al., 2007).

\section{$17 \alpha, 20 \beta$ dihidroxi-4-pregna-3-ona $(17 \alpha, 20 \beta-$ DHP)}

Es una hormona dentro del grupo Esteroides inductores de maduración (MIS por sus siglas en inglés, maturational inductor steroids) que actúa sobre los receptores de membrana de los oocitos activando el factor promotor de maduración en el citoplasma de los oocitos (Nagahama, 1987, 1997).

Ha sido encontrada en Salmóniformes, Characiformes Cipriniformes, Ciprinodontiformes, Siluriformes, Beloniformes, Esociformes, Osteoglosiformes y Clupeiformes (Moreira et al., 2015; Tokarz et al., 2015), su función está asociada a diferentes procesos en la maduración de los productos germinales. En la maduración de los ovocitos estimula la formación del huso, la condensación de la cromatina y la formación del primer cuerpo polar. En los machos estimula la maduración de los espermatocitos al iniciar las divisiones de las células meióticas, además induce la producción del fluido seminal y mejora la motilidad del esperma mediante la alteración del pH (Tokarz et al., 2015).

Estudios en machos de Silúridos asiáticos (Heteropneustes fossilis) durante un ciclo reproductivo (época 
de pre-desove y desove) se encontraron niveles más altos de esta hormona en el testículo durante el pre-desove, en comparación con los bajos niveles en la vesícula seminal durante esta misma época (Chaube, Mishra y Singh, 2018). Esto podría ser explicado por la influencia de esta progestina en los estadios tempranos de espermatogénesis, especialmente al inicio de la meiosis (Amer et al., 2001).

En hembras de Salminus hilarii mantenidas en cautiverio, los niveles de $17 \alpha, 20 \beta$-DHP se incrementaron en la fase previtelogénica y en la fase de maduración avanzada y se mantuvieron durante la regresión (Moreira et al., 2015). Por lo que la incapacidad de estas hembras para desovar, no estaría explicada por la modulación en la producción de este progestágeno, ni por los bajos niveles de síntesis de ARNm de lhb como ha sido mencionado anteriormente (ver subtítulo "Hormona luteinizante").

\section{Conclusión}

Como es posible observar a través de la recopilación de los últimos estudios de esteroides sexuales en peces neotropicales, los patrones de expresión y la función reguladora de estas hormonas en el eje hipotálamo - pituitaria - gónada (HPG) varía en función de las características de las estrategias reproductivas de la especie y las condiciones en las que son mantenidos los ejemplares de estudio (cautiverio, cría en altas o bajas densidades, silvestres, etc.).

Intentar establecer un patrón universal para teleósteos neotropicales es bastante arriesgado. Si bien el esclarecimiento de las rutas de acción de estas hormonas y sus reguladores, se convierte en las bases teóricas para la implementación de herramientas en la producción acuícola, no bastan para explicar las disfunciones reproductivas de estos organismos cuando son sometidos a cautiverio, el gran problema de la acuicultura contemporánea. Por esto, creemos que integrar conocimientos de la historia de vida de las especies, tales como el comportamiento de apareamiento (movimientos, emisión de sonidos, liberación de sustancias químicas, tal vez equivalentes a feromonas en mamíferos, etc.) deben ser probados en un intento de esclarecer los motivos de dichas disfunciones.

Finalmente, consideramos que la investigación desarrollada en las últimas décadas, para el conocimiento detallado de las funciones y regulación de estas hormonas, ha avanzado al punto de producir gonadotropinas recombinantes especie-específicas como el caso de Medaka japonesa (ver Burow et al., 2018) y Carpa común (ver Hollander-Cohen et al., 2018). Sin em- bargo, en especies colombianas de interés comercial como el Bocachico (Prochilodus magdalenae), Sabaleta (Brycon henni), Cachama blanca (Piaractus brachypomus), Cachama negra (Colossoma macropomum), Bagres (Pseudoplatystoma orinocoense, P. magdaleniatum, P. metaense, P. tigrinum), entre otros, estas bases neuroendocrinas de la reproducción siguen siendo desconocidas.

\section{Referencias}

Masafumi A, Shunsuke M, Masayuki L, Shoji K, Noriko A, Kunio Y, Kazuyoshi U, Kazuyoshi T. Novel fish hypothalamic neuropeptides stimulate the release of gonadotrophins and growth hormone from the pituitary of sockeye salmon. I Endocrinol, 2006;188(3):417-423. doi: 10.1677/joe.1.06494.

Mohamed AA, Takeshi M, Chiemi M, Kohei Y. Involvement of Sex Steroid Hormones in the Early Stages of Spermatogenesis in Japanese Huchen (Hucho perryi) ${ }^{1}$. Biol Reprod, 2001; 65(4):1057-1066. doi: 10.1095/biolreprod65.4.1057.

Ando $\mathrm{H}$, Shahjahan $\mathrm{M}$, Kitahashi T. Periodic regulation of expression of genes for kisspeptin, gonadotropin-inhibitory hormone and their receptors in the grass puffer: Implications in seasonal, daily and lunar rhythms of reproduction. Gen Comp Endocrinol, 2018;265:149-153. doi: 10.1016/j.ygcen.2018.04.006.

Andreu-Vieyra CV, Buret AG, Habibi HR. Gronadotropin-releasing hormone induction of apoptosis in the testes of goldfish (Caraasius auratus). Endocrinology, 2005;146(3):1588-1596. doi: 10.1210/en.2004-0818.

Barrero M, Small BC, D'Abramo LR, Hanson LA, Kelly AM. Comparison of estradiol, testosterone, vitellogenin and cathepsin profiles among young adult channel catfish (Ictalurus punctatus) females from four selectively bred strains. Aquaculture, 2007:264(14):390-397. doi: 10.1016/j.aquaculture.2006.12.003.

Biran J, Golan M, Mizrahi N, Ogawa S, Parhar IS, Levavi-Sivan B. LPXRFa, the Piscine Ortholog of $\mathrm{GnIH}$, and LPXRF Receptor Positively Regulate Gonadotropin Secretion in Tilapia (Oreochromis niloticus). Endocrinology, 2014;155(11):4391-4401. doi: 10.1210/en.2013-2047.

Borg B. Androgens in teleost fishes. Comp Biochem Physiol C Pharmacol Toxicol Endocrinol. 1994;109(3):219-245. doi: 10.1016/0742-8413(94)00063-G.

Breton B, Govoroun M, Mikolajczyk T. GTH I and GTH II Secretion Profiles during the Reproductive Cycle in Female Rainbow Trout : Relationship with Pituitary Responsiveness to GnRH-A Stimulation. Gen Comp Endocrinol, 1998;111(1):38-50. doi. org/10.1006/gcen.1998.7088

Burow S, Fontaine R, von Krogh K, Mayer I, Nourizadeh-Lillabadi R, Hollander-Cohen L, et al. Medaka follicle-stimulating hormone (Fsh) and luteinizing hormone (Lh): Developmental profiles of pituitary protein and gene expression levels. Gen Comp Endocrinol, 2018;272:93-108. doi: 10.1016/j.ygcen.2018.12.006.

Cavaco JE, Bogerd J, Goos H, Schulz RW. Testosterone Inhibits 11-Ketotestosterone-Induced Spermatogenesis in African Cat- 
fish (Clarias gariepinus) ${ }^{1}$. Biol Reprod, 2001;65(6):1807-1812. doi: 10.1095/biolreprod65.6.1807.

Chaube R, Mishra S, Singh RK. A comparison of steroid profiles in the testis and seminal vesicle of the catfish (Heteropneustes fossilis). Theriogenology, 2018; 105:90-96. doi: 10.1016/j.theriogenology.2017.09.010.

Chowdhury VS, Ubuka T, Osugi T, Shimura T, Tsutsui K. Identification, localization and expression of LPXRFamide peptides, and melatonin-dependent induction of their precursor mRNA in the newt brain. J Endocrinol, 2011;209(2):211-220. doi: 10.1530/ JOE-10-0494.

Ciccone NA, Dunn IC, Boswell T, Tsutsui K, Ubuka T, Ukena K, Sharp PJ. Gonadotrophin Inhibitory Hormone Depresses Gonadotrophin alpha and Follicle-Stimulating Hormone beta Subunit Expression in the Pituitary of the Domestic Chicken", J Neuroendocrinol, 2004;16(12):999-1006. doi: 10.1111/j.13652826.2005.01260.x.

Devlin RH, Nagahama Y. Sex determination and sex differentiation in fish: an overview of genetic, physiological, and environmental influences. Aquaculture, 2002;208(3-4):191-364. doi: 10.1016/S0044-8486(02)00057-1.

Felip A, Zanuy S, Pineda R, Pinilla L, Carrillo M, Tena-Sempere M, Gómez A. Evidence for two distinct KiSS genes in non-placental vertebrates that encode kisspeptins with different gonadotropin-releasing activities in fish and mammals. Mol Cell Endocrinol, 2009;312(1-2):61-71. doi: 10.1016/j.mce.2008.11.017.

Hollander-Cohen, L. et al. Characterization of carp gonadotropins: Structure, annual profile, and carp and zebrafish pituitary topographic organization. Gen Comp Endocrinol. 2018;264:28-38. doi: 10.1016/j.ygcen.2017.11.022.

Honji RM, Caneppele D, Pandolfi M, Lo Nostro FL, GuimarãesMoreiraa R. Characterization of the gonadotropin-releasing hormone system in the Neotropical teleost, Steindachneridion parahybae during the annual reproductive cycle in captivity. Gen Comp Endocrinol, 2019;273:73-85.doi: 10.1016/j.ygcen.2018.05.007.

Hoskins LJ, Xu M, Volkoff $\mathrm{H}$. Interactions between gonadotropin-releasing hormone $(\mathrm{GnRH})$ and orexin in the regulation of feeding and reproduction in goldfish (Carassius auratus). Horm Behav, 2008;54(3):379-385. doi: 10.1016/j.yhbeh.2008.04.011.

Hou ZS, Wen HS, Li JF, He F, Li Y, Tao YX. Hypothalamus-pituitarygonad axis of rainbow trout (Oncorhynchus mykiss) during early ovarian development and under dense rearing condition. Gen Comp Endocrinol, 2016;236:131-138. doi: 10.1016/j.ygcen.2016.07.011.

de Jesus LWO, Bogerd J, Vieceli FM, Branco GS, Camargo MP, Cassel M, Moreira RG, et al. Gonadotropin subunits of the characiform Astyanax altiparanae: Molecular characterization, spatiotemporal expression and their possible role on female reproductive dysfunction in captivity. Gen Comp Endocrinol, 2017;246:150-163. doi: 10.1016/j.ygcen.2016.12.004.

Kajimura S. et al. cDNA Cloning of Two Gonadotropin $\beta$ Subunits $(\mathrm{GTH}-\mathrm{\beta}$ and $-\| \beta)$ and Their Expression Profiles during Gametogenesis in the Japanese Flounder (Paralichthys olivaceus). Gen Comp Endocrinol, 2001;122(2):117-129. doi: 10.1006/ GCEN.2000.7610.
Karigo T, Oka Y. Neurobiological Study of Fish Brains Gives Insights into the Nature of Gonadotropin-Releasing Hormone 1-3 Neurons. Front Endocrinol, 2013;4:177. doi: 10.3389/fendo.2013.00177.

Landines MA, Prieto CA, Rodriguez L, Rosado R. Perfil de esteroides sexuales del capitán de la sabana (Eremophilus mutisii) durante un ciclo hidrológico completo. Rev udca Actual Divulg Cient, 2017;20(1):43-50.doi: <http://www.scielo.org.co/scielo. php?script=sci_arttext\&pid=S0123-42262017000100006\&lng $=$ en\&nrm $=$ iso $>$.

Levavi-Sivan B, Bogerd J, Mananos EL, Gomez A, Lareyre JJ. Perspectives on fish gonadotropins and their receptors. Gen Comp Endocrinol, 2010;165(3):412-437. doi: 10.1016/J.YGCEN.2009.07.019.

Lubzens E, Young G, Bobe J, Cerdà J. Oogenesis in teleosts: How fish eggs are formed. Gen Comp Endocrinol, 2010;165(3):367389. doi: 10.1016/J.YGCEN.2009.05.022.

Maitra SK, Hattoraj A, Mukherjee S, Moniruzzaman M. Melatonin: A potent candidate in the regulation of fish oocyte growth and maturation. Gen Comp Endocrinol, 2013;181(1):215-222. doi: 10.1016/j.ygcen.2012.09.015.

Mateos J, Mañanósa E, Martínez-Rodríguez G, Carrillo M, Querat B, Zanuy S. Molecular characterization of sea bass gonadotropin subunits $(\alpha, \mathrm{FSH} \beta$, and $\mathrm{LH} \beta)$ and their expression during the reproductive cycle. Gen Comp Endocrinol, 2003;133(2):216-232. doi: 10.1016/S0016-6480(03)00164-3.

Matsuda K, Nakamura K, Shimakura SI, Miura T, Kageyama H, Uchiyama M, Shioda S, Ando H. Inhibitory effect of chicken gonadotropin-releasing hormone II on food intake in the goldfish, Carassius auratus. Horm Behav, 2008;54(1):83-89. doi: 10.1016/j.yhbeh.2008.01.011.

Melamed P, Sherwood N. (2005) Hormones and Their Receptors in Fish Reproduction. WORLD SCIENTIFIC (Molecular Aspects of Fish \& Marine Biology). doi: 10.1142/5533.

Melo MC, Dijk P, Andersson E, Nilsen T, Fjelldal P, Male R, et al. Androgens directly stimulate spermatogonial differentiation in juvenile Atlantic salmon (Salmo salar). Gen Comp Endocrinol, 2015;211:52-61. doi: 10.1016/j.ygcen.2014.11.015.

Miranda LA, Chalde T, Elisio M, Strüssmann CA. Effects of global warming on fish reproductive endocrine axis, with special emphasis in pejerrey Odontesthes bonariensis. Gen Comp Endocrinol, 2013;192:45-54. doi: 10.1016/j.ygcen.2013.02.034.

Miura T, Miura C, Ohta T, Nader MR, Todo T, Yamauchi K. Estradiol$17 \beta$ Stimulates the Renewal of Spermatogonial Stem Cells in Males. Biochem Biophys Res Commun, 1999;264(1):230-234. doi: 10.1006/BBRC.1999.1494.

Mommsen TP, Vijayan MM, Moon TW. Cortisol in teleosts: dynamics, mechanisms of action, and metabolic regulation. Rev Fish Biol Fisher, 1999;9(3):211-268. doi: 10.1023/A:1008924418720.

Moreira RG, Honji RM, Melo RG, Narcizo AM, Amaral JS, Araújo RC, Hilsdorf AW. The involvement of gonadotropins and gonadal steroids in the ovulatory dysfunction of the potamodromous Salminus hilarii (Teleostei: Characidae) in captivity. Fish Physiol Biochem, 2015;41(6):1435-1447. doi: 10.1007/s10695015-0097-y. 
Nagahama Y. 7 alpha, 20 beta-Dihydroxy-4-pregnen-3-one: A Teleost Maturation-Inducing Hormone. Dev Growth Differ. 1987;29:1-12.

Nagahama Y. Endocrine regulation of gametogenesis in fish. Int I Dev Biol, 1994;38(2):217-229. doi: doi=7981031.

Nagahama Y. 17 $\alpha, 20 \beta$-Dihydroxy-4-pregnen-3-one, a maturation-inducing hormone in fish oocytes: Mechanisms of synthesis and action. Steroids. Elsevier, 1997;62(1):190-196. doi: 10.1016/ S0039-128X(96)00180-8.

Nagahama Y, Yamashita M. Regulation of oocyte maturation in fish. Dev Growth Differ, 2008;50(suppl1):S195-219. doi: 10.1111/j.1440-169X.2008.01019.x.

Nyuji M, Hamada K, Kazeto Y, Mekuchi M, Gen K, Soyano K, Okuzawa K. Photoperiodic regulation of plasma gonadotropin levels in previtellogenic greater amberjack (Seriola dumerili). Gen Comp Endocrinol, 2018;269:149-155. doi: 10.1016/j.ygcen.2018.09.007.

Okubo K, Nagahama Y. Structural and functional evolution of gonadotropin-releasing hormone in vertebrates. Acta Physiologica, 2008;193(1):3-15. doi: 10.1111/j.1748-1716.2008.01832.x.

Park JW, Jin YH, Oh SY, Kwon JY. Kisspeptin2 stimulates the HPG axis in immature Nile tilapia (Oreochromis niloticus). Comp Biochem Physiol Part B: Biochem Mol Biol, Biochemistry and Molecular Biology, 2016;202:31-38. doi: 10.1016/j. cbpb.2016.07.009.

Passini G, Carvalho CVA, Carneiro-Sterzelecki F, Francisco-Baloia M, Ronzani-Cerqueira V. Spermatogenesis and steroid hormone profile in puberty of laboratory-reared common snook (Centropomus undecimalis). Aquaculture, 2019;500:622-630. doi: 10.1016/j.aquaculture.2018.10.031.

Peng C, Chang JP, Yu KL, Wong AO, Van-Goor F, Peter RE, Rivier JE. Neuropeptide-Y stimulates growth hormone and gonadotropin-II secretion in the goldfish pituitary: involvement of both presynaptic and pituitary cell actions. Endocrinology, 1993;132(4):1820-1829. doi: 10.1210/endo.132.4.8462479.

Peng W, Cao M, Chen J, Li Y, Wang Y, Zhu Z, Hu W. GnlH plays a negative role in regulating $\mathrm{GtH}$ expression in the common carp, Cyprinus carpio L. Gen Comp Endocrinol, 2016;235:18-28. doi: 10.1016/j.ygcen.2016.06.001.

Planas JV, Swanson P. Maturation-Associated Changes in the Response of the Salmon Testis to the Steroidogenic Actions of Gonadotropins (GTH I and GTH II ) In Vitro. Biol Reprod, 1995;52(3):697-704. doi:10.1095/biolreprod52.3.697

Qi X, Zhou W, Li S, Lu D, Yi S, Xie R, Liu X, Zhang Y, Lin H. “Evidences for the regulation of GnRH and GTH expression by GnlH in the goldfish, Carassius auratus", Mol Cell Endocrinol, 2013;366(1):9-20. doi: 10.1016/j.mce.2012.11.001.

Rather MA, Bhat IA, Gireesh-Babu P, Chaudhari A, Sundaray JK, Sharma R. Molecular characterization of kisspeptin gene and effect of nano-encapsulted kisspeptin-10 on reproductive maturation in Catla catla. Domest Anim Endocrinol, 2016;56:36-47. doi: 10.1016/j.domaniend.2016.01.005.

Rocha MJ, Arukwe A, Kapoor BG. (2008) Fish reproduction. 1st ed. Science Publishers. Pp.632.
Saborido-Rey F. (2008) Ecología de la reproducción y potencial reproductivo en las poblaciones de peces marinos. Instituto de Investigaciones Marinas (CSIC) Universidad de Vigo. Pp.71.

Sawada K, Ukena A, Satake H, Iwakoshi E, Minakata H, Tsutsui K. "Novel fish hypothalamic neuropeptide". Eur J Biochem, 2002;269(24):6000-6008. doi: 10.1046/j.14321033.2002.03351.x

Schulz RW, de França LR, Lareyre JJ, Le Gac F, Chiarini-Garcia H, Nobrega RH, Miura T. Spermatogenesis in fish. Gen Comp Endocrinol, 2010;165(3):390-411. doi: 10.1016/j.ygcen.2009.02.013.

Swanson P, Dickey JT, Campbell B. Biochemistry and physiology of fish gonadotropins. Fish Physiol Biochem, 2003;28:53-59.

Tena-Sempere M, Felip A, Gómez A, Zanuy S, Carrillo M. Comparative insights of the kisspeptin/kisspeptin receptor system: Lessons from non-mammalian vertebrates". Gen Comp Endocrinol, 2012;175(2):234-243. doi: 10.1016/j.ygcen.2011.11.015.

Thorson JF, Prezotto LD, Cardoso RC, Sharpton SM, Edwards JF, et al. (2014) "Hypothalamic Distribution, Adenohypophyseal Receptor Expression, and Ligand Functionality of RFamide-Related Peptide 3 in the Mare During the Breeding and Nonbreeding Seasons1". Biology of Reproduction, 2014;90(2):1-9. doi: 10.1095/biolreprod.113.112185.

Tokarz J, Möller G, de Angelis MH, Adamski J. "Zebrafish and steroids: What do we know and what do we need to know?". J Steroid Biochem Mol Biol, 2013;137:165-173. doi: 10.1016/j. jsbmb.2013.01.003.

Tokarz J, Möller G1, Hrabě de Angelis M, Adamski J. Steroids in teleost fishes: A functional point of view. Steroids, 2015;103:123144. doi: 10.1016/j.steroids.2015.06.011.

Tsutsui K, Saigoh E, Ukena K, Teranishi H, Fujisawa Y, Kikuchi M, et al. A Novel Avian Hypothalamic Peptide Inhibiting Gonadotropin Release. Biochem Biophys Res Commun, 2000;275(2):661-667. doi: 10.1006/BBRC.2000.3350.

Tsutsui K. A new key neurohormone controlling reproduction, gonadotropin-inhibitory hormone $(\mathrm{GnlH})$ : Biosynthesis, mode of action and functional significance. Progress in Neurobiology, 2009;88(1):76-88. doi: 10.1016/j.pneurobio.2009.02.003.

Tsutsui K, Osugi T, Son YL, Ubuka T. Review: Structure, function and evolution of GnlH. Gen Comp Endocrinol, 2018;264:4857. doi: 10.1016/j.ygcen.2017.07.024.

Ubuka T, Bentley GE, Ukena K, Wingfield JC, Tsutsui K. Melatonin induces the expression of gonadotropin-inhibitory hormone in the avian brain. Proc Natl Acad Sci USA, 2005;102(8):30523057. doi: 10.1073/PNAS.0403840102.

Ubuka T, Morgan K, Pawson AJ, Osugi T, Chowdhury VS, Minakata $\mathrm{H}$, et al. Identification of Human GnlH Homologs, RFRP-1 and RFRP-3, and the Cognate Receptor, GPR147 in the Human Hypothalamic Pituitary Axis. PLoS One, 2009;4(12):e8400. doi: 10.1371/journal.pone.0008400.

Ubuka T, Son YL, Tsutsui K. Molecular, cellular, morphological, physiological and behavioral aspects of gonadotropin-inhibitory hormone. Gen Comp Endocrinol, 2016;227:27-50. doi: 10.1016/j.ygcen.2015.09.009. 
Ukena K, Iwakoshi-Ukena E, Osugi T, Tsutsui K. Identification and localization of gonadotropin-inhibitory hormone $(\mathrm{GnlH})$ orthologs in the hypothalamus of the red-eared slider turtle, Trachemys scripta elegans. Gen Comp Endocrinol, 2016;227:69-76. doi: 10.1016/j.ygcen.2015.06.009.

Valdebenito I, Paiva L, Berland M. Atresia folicular en peces teleósteos : una revisión. Arch Med Vet, 2011;43(1):11-25. doi. org/10.4067/S0301-732X2011000100003

Volkoff $\mathrm{H}$. The Neuroendocrine Regulation of Food Intake in Fish: A Review of Current Knowledge. Front Neurosci, 2016;10:540. doi: 10.3389/fnins.2016.00540.

Wu C, Patiño R, Davis KB, Chang X. Localization of estrogen receptor $\alpha$ and $\beta$ RNA in germinal and nongerminal epithelia of the channel catfish testis. Gen Comp Endocrinol, 2001;124(1):1220. doi: 10.1006/gcen.2001.7668.

Wylie MJ, Setiawan AN, Irvine GW, Symonds JE, Elizur A, Lokman PM. Effects of neuropeptides and sex steroids on the pituitarygonadal axis of pre-pubertal F1 wreckfish (hāpuku) Polyprion oxygeneios in vivo: Evidence of inhibitory effects of androgens. Gen Comp Endocrinol, 2018;257:113-121. doi: 10.1016/j.ygcen.2017.08.018.
Yaron Z, Gur G, Melamed P, Rosenfeld H, Elizur A, Levavi-Sivan B. Regulation of fish gonadotropins. Int Rev Cytol, 2003;225:131185. doi.org/10.1016/S0074-7696(05)25004-0

Yu KL, Peter RE. Alterations in gonadotropin-releasing hormone immunoactivities in discrete brain areas of male goldfish during spawning behavior. Brain Research, 1990;512(1):89-94. doi: 10.1016/0006-8993(90)91174-F.

Yu KL, Rosenblum PM, Peter RE. In vitro release of gonadotropin-releasing hormone from the brain preoptic-anterior hypothalamic region and pituitary of female goldfish. Gen Comp Endocrinol, 1991;81(2):256-267. doi: 10.1016/0016-6480(91)90010-4.

Zhang Y, Li S, Liu Y, Lu D, Chen H, Huang X, et al. Structural diversity of the gnih/gnih receptor system in teleost: Its involvement in early development and the negative control of $\mathrm{LH}$ release. Peptides, 2010;31(6):1034-1043. doi: 10.1016/j.peptides.2010.03.003.

Zohar Y, Muñoz-Cueto JA, Elizur A, Kah O. Neuroendocrinology of reproduction in teleost fish. Gen Comp Endocrinol, 2010;165(3):438-455. doi: 10.1016/j.ygcen.2009.04.017.

Mónica Nieto: https://orcid.org/0000-0001-7241-6089 José Rodríguez: https://orcid.org/0000-0002-6735-3885 Agustín Góngora: https://orcid.org/0000-0002-2915-683X 